\title{
Research on the Regulation and Control Technology of High Load Energy Load in Response to Load Loss Demand of Power Network
}

\author{
Yong Yang a,*, Jingjing Zheng, Fuchao Liu and Jing Peng \\ Gansu Electric Power Research Institute Lanzhou 730070.China \\ State Grid Gansu Intergrated Enegy Service Co.,Ltd. Lanzhou 730070.China \\ ay8801@126.com \\ *Corresponding author
}

Keywords: High-energy, the peak modulation, Reduce the loss.

\begin{abstract}
This paper, through the study of high load characteristic of peak load and high load, regulation and control mode of conventional power and new energy hybrid system, the long time scale load in peakload regulation strategy, short time scale load in peak modulation strategy, explores high load in power system charge source coordination loss reduction and new model and solves the new energy power generation and provides a new way for network environment under load source interaction loss reduction.
\end{abstract}

\section{Introduction}

Surge in the loss of power transmission network, conventional power peak capacity is insufficient, new energy sources send blocked a series of bottleneck, simply through the source to source and source coordination mode of operation has been unable to meet the needs of the development of large-scale new energy base, need to open up new ideas, to discover new patterns, to seek a new method to solve the base of new energy development and energy conservation power loss reduction of two-way contradiction. In this paper, we study high load can load adaptive grid loss demand regulation and control technology. To explore the high load can load in power system charge source coordination loss reduction and new model and solve the new energy power generation and network environment under load source interaction loss reduction provides a new way [1] .

\section{The Characteristics of High Energy Load Peak Load}

The peak load regulation characteristics analysis of the load adjustment ability, capacity constraints, considering the economic load, delay the control commands. Load control, participate in peaking of the ultimate goal is the load with similar to conventional thermal power units of the ability to regulate. Thermal power units can be enough to ensure the minimum output based on continuous adjustment of its output, but can't bear to volatility.

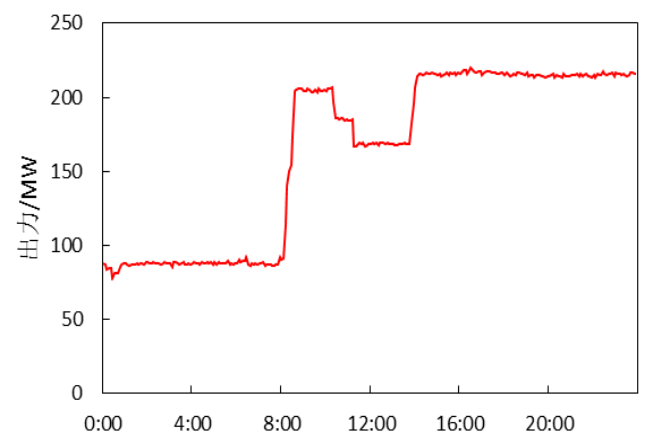

Fig. 1 The daily output curve of Jingyuan power plant 
Figure 1 is Jingyuan power plant, the output curve. Thermal power plant output curve was the ladder - shaped, indicating that the thermal power in the adjustment process in addition to meet the requirements of climbing rate, when after a major climbing shall be maintained for a period of time, otherwise the frequent adjustment the normal operation of the impact of thermal power unit.

(1) The transfer of peaking capacity and load characteristics

Load transfer is for those who is not a kind of load, by adjusting the production time, change load curve of the peak load regulating units. In practical production, the load transfer, transfer time is affected by the timing of production. Mainly considered the supply of raw materials, equipment utilization factor in operations research, such as after the completion of the task of the upper reaches of the equipment needs certain material transfer or wait for time to start the task of the downstream equipment; the task on the same device need after the end of a task to open a mission to ensure that equipment exclusive. Due to production time transfer across time adjust fire than the motor group flexible [2].

(2) Peak load regulation ability and characteristics of continuous

Continuous adjustment of the peaking capacity of load is similar with that of thermal power units. In load adjustment range within, can bear certain ramp rate, but not frequent adjustment, because part of the high precision of production equipment, component compression resistant shock ability limited, its peaking characteristics are certainly inferior to fire motor group. After an uphill shall stable production for a long time and adjusting time shoulds not be too long, when must by the regulation gradually returned to the initial operation state. As the load equivalent owned power plant load characteristics is similar with that of the conventional thermal power units.

(3) The interruptible load characteristics and load capacity

Part load has the advantages of simple equipment, power loss, and consumption, such as smelting, cement and other load. Continuous production of cement companies in order to produce efficiency, but not entirely because of the technical limitations of the process, interruptible load because of its simple equipment, peaking capacity is much better than that of conventional thermal power units, a regulation to produce step, obviously alleviate shortage of power system.

(4) Peak method load in multi time scales

Load transfer, continuous load regulation and interruptible load is the peak method on different time scales. The load transfer method is peaking long time, it is necessary to determine its transfer strategy in the day before, in the days according to more accurate prediction of wind power and load prediction load transfer to run load in the power plant. If the boot can be continuous, according to the arrangement of the power grid, it also requires long time scale to arrange the opening machine, you should adjust the way only as a short time scale. Although the interruptible load in power consumption and has important effect on system peak load, but not the main interruptible load dispatching mode should be considered is the standby system, only take effect in power grid power shortage situation.

In the short time scale, should give full play to have continuous adjusting load adjustment ability, consumptive renewable energy increment. Interruptible load should also be disruption in the power grid power shortage situation. But in specific regulation should be with conventional power grid peak shaving method reasonably together.

\section{Control Modes of High Load, Conventional Power and New Energy Hybrid System}

High load can be seen as the power supply system for "standby" or "negative standby" in Dutch network coordination of source control system can be regarded as a "virtual power". Therefore, high load, the conventional power and renewable energy hybrid system can adopt similar stream combined power generation system of the mode of regulation. In the coordinated operation of the hybrid system, you first need to determine the operating system to achieve the goal, the realization of the control objectives, will lead to different mode of system regulation. 
The control mode of the combined system is smooth power output mode and tracking scheme output mode and cut a peak to fill valley model [3].

(1) Smooth power output mode

Smooth power output mode including inhibition of wind power short-term fluctuations and long-term fluctuations, thereby increasing the stability of wind power output. In this mode, we should according to the wind speed forecast data, the calculated total output power curve of wind power, based on this formulation high load peak load regulation strategy. Smooth power output mode mainly to smooth the output of short-term wind power output fluctuations, thus increasing the stability of power output of the system, the desired configuration of regulatory capacity and system output power needed to reach the degree of smoothing. Under normal circumstances, the United System of energy dispatch strategies follow the following principles:

1) Participate in the industry of wind power wind power wind power in total power output is large, an increase of high load to industrial production load. In the total power output is small, to reduce the energy industry production load, and should try to reduce the high load can load adjustment frequency.

2) under high load can load regulating capacity and renewable energy output prediction curve, the reasonable selection of high load can load regulation the start time, duration, etc., as far as possible for a long time to maintain a relatively stable total output power.

3) in the total output of small, and high load can load has certain wealthy adjustable capacity, the capacity of system load cut peak and fill valley.

(2) Tracking scheme output mode

Track the generation scheduling model is according to the planning system output curves, in the conventional unit does not meet the regulation requirements regulating high load can load process, make the actual output power of the hybrid system as close as possible to the scheduled output, thereby increasing the certainty of the power of the wind power output. Plan output curve can be according to the wind speed forecasting that power prediction output curve, can also be according to the plan formulated by the share of the United System of regional power load characteristics output curve. Under normal circumstances, follow the following energy scheduling strategy:

1) As far as possible absorb wind power, namely, let wind power to meet the needs of load, if there is a rich wind energy is adjusted high load can be load consumptive;

2) If possible use of wind power generation still cannot satisfy the load needs, at this time if the high load can load regulating capacity adequacy, consider adjusting high load can meet the load of a load;

3) If the plan output curve is beyond the scope of regulation and control of hybrid systems, as far as possible to control output power of the hybrid system close to the planned output curve, but does not require actual output power and output is exactly the same.

Further subdivision, tracking the typical model of the output of the following two types:

1) In wind power output forecasting curve for plan curve. In this case the high load capacity configuration and size adjustable wind power output accuracy of the predicted values, the smaller the prediction error needed regulation capacity is small. This model can eliminate the influence of the uncertainty of wind power on the grid, but for fluctuation suppression ability is weak, applicable to the ability of the grid. The 2 peak area) by the system load characteristic allocation plan output curve. The condition of high load characteristics of the adjustable load capacity configuration size and area load. Generally speaking, matching is poor between the wind power output power and load in northern China, power shortage index is also serious.

(3) Peak shaving mode

In this mode, high energy load regulation load is relatively fixed, usually according to the peaks and valleys of the regional wind power output power as high load regulation of the switching point. In general, follow the energy scheduling strategy is as follows:

1) To reach the peak of wind power at night, high energy industrial production increased load and wind power output to absorb surplus power and energy; 
2) In the daytime wind fall into the trough period, high energy consuming industry to bring down the production capacity, in order to achieve peak wind power transfer, partly offset by anti wind power peaking characteristics;

3) Of high load regulation, as far as possible with conventional to ensure the power fluctuation of wind power output in the acceptable range. This model according to the characteristics of peak and valley wind power output, with adjusting the conventional power supply, the output peak in the wind Enable high load can be load consumptive excess energy, in the wind power output trough cut part of the high load to reduce the power output of wind electricity peak valley difference, the theory can be mixed system adjustment of the output curve is a straight line [4].

\section{Long Time Scale Load Cycling Strategy}

On long time scales, recently most of the plan according to the network status and historical mode of operation, based on expert experience, combined with heuristic method according to certain logical calculation form. Such as in equilibrium power generation dispatch mode, consider annual contract quantity, consumption and other factors have been completed, for each unit in the planning, boot program and expected energy.

Load to load transfer under the condition of input to actual data in this case, can be based on wind power prediction value to determine the transfer of load regulation. As shown in Figure 2 is shown in nine consecutive days of wind power. If the load transfer in different day transfer (such as silicon carbide, iron alloy load in electricity production, electricity, no electric stop production), should be December 12, 15, 16, 17 and 18 days of production, which can reduce the equivalent load peak valley difference. If only in the days of the transfer, can be on the 14th afternoon moved to the wee hours of the morning wind production time, reduce the peak valley difference.

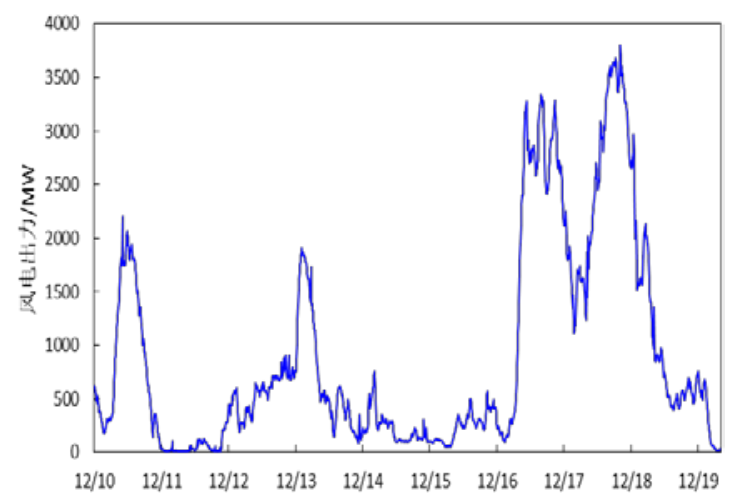

Fig. 2 12, 11, 19, the wind power curve

Transfer load before the stage of peak shaving method are summarized as follows: (1) on the basis of monthly wind power prediction, the arrangement has a longer production of load capacity of load transfer, more load arrangement to wind TVU daily; (2) in the days of time scale, according to the wind and load predictions, will be able to transfer the load from the wind peak load period transfer to the wind valley load stage; (3) according to the forecast, wind power, days rolling revision transfer load in the beginning of time.

\section{Short Time Scale in Peak Load Strategy}

Hydro thermal power peaking characteristics of the load peaking characteristics involved. For load, in its not involved in the regulation of, has been close to the production in the rated condition. When the application of continuous adjustment ability, especially to improve the load increment in the process of tracking renewable energy. The increased load, long time will continue to electric equipment adversely. Under normal circumstances, to meet their own load 
power plant enterprise, this is the economic means for the enterprise. If the enterprise owned power plant down power supply load, on the one hand, rising energy consumption per unit of power plant, on the other hand, the purchase of electricity enterprises from the grid, increase the cost of production. And improve the reliability of power supply is the object of power grid enterprises pursue, so in the case of power shortage, don't interrupt load regulation. To sum up, in the normal regulation of power grid Peak capacity to meet peak demand, without application of load in load. But when the scenery TV during the period and the lack of effective peak shaving methods provide the Internet space, abandoned wind abandoned light will cause the waste of energy under the premise, applied load adjustable peaking characteristics can reduce the abandoned wind light; when the power shortage of interruptible load to stop power supply to ensure power supply to a level load [5] .

In view of this, we should first determine the continuous adjustment of the load is qualified to participate in the power system peak load conditions. According to the abandoned wind, abandoned light principle, abandoned wind, abandoned light occurs, power system equivalent load is small, conventional power (hydropower, thermal power) depress production load, when meet the wind farm power less than the predicted power, predict wind power continues to increase and conventional power close to the minimum capacity of production shall be enabled continuously adjustable load in peak shaving. Similarly, when power system into a state of power shortage, the conventional hydrothermal power close to the rated power, the wind photoelectric predict further decreased when shall implementation of interruptible load participating in shifting peak load.

In view of this, we should first determine the continuous adjustment of the load is qualified to participate in the power system peak load conditions. According to the abandoned wind, abandoned light principle, abandoned wind, abandoned light occurs, power system equivalent load is small, conventional power (hydropower, thermal power) depress production load, when meet the wind farm power less than the predicted power, predict wind power continues to increase and conventional power close to the minimum capacity of production shall be enabled continuously adjustable load in peak shaving. Similarly, when power system into a state of power shortage, the conventional hydrothermal power close to the rated power, the wind photoelectric predict further decreased when shall implementation of interruptible load participating in shifting peak load.

Table 1 Peak availability degree and for example

\begin{tabular}{|c|c|c|}
\hline $\begin{array}{c}\text { Peak } \\
\text { value }\end{array}$ & instruction & example \\
\hline 1 & $\begin{array}{c}\text { The peak as the main purpose of existence, } \\
\text { have a strong willingness to peak }\end{array}$ & $\begin{array}{c}\text { Energy storage, pumped storage } \\
\text { power station }\end{array}$ \\
\hline 2 & $\begin{array}{c}\text { In load does not affect the economics of } \\
\text { electricity }\end{array}$ & $\begin{array}{c}\text { Hydropower station with hours to } \\
\text { adjust the above scale }\end{array}$ \\
\hline 3 & $\begin{array}{c}\text { In the peak of economic effects on hair not } \\
\text { electricity }\end{array}$ & $\begin{array}{c}\text { Medium temperature and medium } \\
\text { temperature and medium } \\
\text { temperature thermal power unit }\end{array}$ \\
\hline 4 & $\begin{array}{c}\text { Peaking to avoid loss of power system with } \\
\text { power greater impact, but for, with response } \\
\text { to grid scheduling, participate in peaking } \\
\text { will }\end{array}$ & $\begin{array}{c}\text { Can quickly start and stop the } \\
\text { thermal power unit, the high load } \\
\text { can be regulated by the load of the } \\
\text { power plant }\end{array}$ \\
\hline 5 & $\begin{array}{c}\text { The peaking cost is very large, should be } \\
\text { adjusted to other power / load to ensure their } \\
\text { normal operation }\end{array}$ & $\begin{array}{c}\text { First order load, runoff type } \\
\text { hydropower station, heating power } \\
\text { station, nuclear power station }\end{array}$ \\
\hline
\end{tabular}


Rather an ability to track peaking resources regulating the tracking ability, in a cycle power variable amplitude and speed regulation. Thermal power units bear a climbing need stable running a period of time, the hydropower generating units can be continued to change and pumped storage can continue to change, high load can load the self tracking capability of the power plant is similar with that of thermal power units, and the continuous adjustment of load tracking ability weaker. Table 2 depicts the different tracking ability and examples [6].

Table 2 The degree of different tracking ability and examples

\begin{tabular}{|c|c|c|}
\hline tractability & instruction & example \\
\hline 1 & Continuous tracking & Hydroelectric generating units \\
\hline 2 & Step tracking & $\begin{array}{c}\text { Thermal power unit, self owned } \\
\text { power plant }\end{array}$ \\
\hline 3 & $\begin{array}{c}\text { Step track and adjust a need to } \\
\text { continue for a long time, cannot } \\
\text { be repeated several steps to adjust }\end{array}$ & High load energy load \\
\hline 4 & Cannot load & $\begin{array}{c}\text { First order load, runoff type } \\
\text { hydropower station, heating power } \\
\text { station, nuclear power station }\end{array}$ \\
\hline
\end{tabular}

Accordingly, can draw the actual order of peak shaving and coordination relations, as shown in Table 3 (of which first is the peak strategy of long time scale).

Table 3 The actual peak order and coordination relations

\begin{tabular}{|c|c|c|c|}
\hline $\begin{array}{l}\text { Peak } \\
\text { order }\end{array}$ & Peak intention & Peak form & Peak effect \\
\hline 1 & $\begin{array}{l}\text { To reduce the peak valley } \\
\text { difference, so that the electric } \\
\text { power system to deal with } \\
\text { abandoned wind light and } \\
\text { electricity shortage }\end{array}$ & $\begin{array}{l}\text { Move the load } \\
\text { from the } \\
\text { equivalent load } \\
\text { peak to the valley. }\end{array}$ & $\begin{array}{l}\text { Reduced the equivalent load } \\
\text { peak and valley difference }\end{array}$ \\
\hline 2 & $\begin{array}{l}\text { Continuous adjustment } \\
\text { ability of application of high } \\
\text { load, consumptive renewable } \\
\text { energy increment }\end{array}$ & step change & $\begin{array}{l}\text { The main consumption of } \\
\text { renewable energy increment, } \\
\text { reduce the abandoned wind, } \\
\text { abandoned light }\end{array}$ \\
\hline 3 & $\begin{array}{l}\text { Application of high load of } \\
\text { power plant, consumptive } \\
\text { renewable energy increment }\end{array}$ & Step change & $\begin{array}{l}\text { The main consumption of } \\
\text { renewable energy increment, } \\
\text { reduce the abandoned wind, } \\
\text { abandoned light }\end{array}$ \\
\hline 4 & $\begin{array}{l}\text { Thermal power peaking, bear } \\
\text { the main task of adjusting the } \\
\text { peak }\end{array}$ & $\begin{array}{l}\text { Ladder type } \\
\text { change, change } \\
\text { amplitude and } \\
\text { frequency is small }\end{array}$ & $\begin{array}{l}\text { Basically completed peaking } \\
\text { task (in thermal power } \\
\text { accounted for relatively large } \\
\text { power system) or completed } \\
\text { part of the task of adjusting } \\
\text { the peak (in thermal power } \\
\text { accounted for general power } \\
\text { system) }\end{array}$ \\
\hline 5 & $\begin{array}{l}\text { Hydropower units peaking, } \\
\text { bear the main task of } \\
\text { adjusting the peak }\end{array}$ & $\begin{array}{l}\text { Continuous } \\
\text { change, change } \\
\text { range and } \\
\text { frequenc }\end{array}$ & $\begin{array}{l}\text { Complete the task of } \\
\text { adjusting the peak }\end{array}$ \\
\hline
\end{tabular}

Based on the above analysis, can get the high load in the short time scales peak strategy:

(1) Determine the hydro thermal power peaking capacity is exhausted, if prepared by high 
energy load peak load;

(2) Lack of peaking capacity in hydrothermal condition, on the basis of wind power forecasting, load forecasting, calculating the residual after the peak demand of Hydropower Participation; or lack of peaking capacity in hydro thermal power, hydro thermal power calculation after adjustment need cutting load;

(3) High load in the peak, peak to peak demand (2) to calculate the plant load itself as sequence distribution of peak shaving tasks. The task in the constraint and load power plant itself under the ramp rate, released in ladder form. Power plant capable of multiple step change, the change of the load limited number;

(4) According to the interruptible load shedding load cost and capacity of selected cutting load sequence and cut implementation bearing;

(5) consider (3) or (4) execution, adjustment of hydrothermal power output, power output adjustment with load adjustment of intent, hydropower to meet all other peaking remaining peak demand, mainly fluctuation of peak demand;

(6) when the system is restored to the hydro thermal power to complete the peaking of state, and gradually restore the high load can be the initial state of the load.

\section{Conclusions}

Through to high load characteristic of peak load and high load, regulation and control mode of conventional power and new energy hybrid system, the long time scale load in peak load regulation strategy and short time scales, the load in peak regulation strategies of technology research, draw the different regulating strategies, for high load to participate in the coordination and control of load network source coordinated control and provide a theoretical basis.

\section{References}

[1] WEN Jing, Liu Wenying, Xie Chang, Wang Weizhou. And the source of electric power system of wind power consumptive benefit charge coordination of two layer optimization model for [J]. Electrotechnical journal. 2015 (08) had.

[2] Bo, Yang Yongqi, Jinhui, Zeng Ming, Ouyang on, Li Chen. The prospect of [J]. automation of electric power systems and key issues for future research response the demand of the new energy power system side. 2015 (17).

[3] sun min, Dou Xiaobo, Shen Yang, Zeng Wei, He Wei. Micro grid before the economic dispatch method [J]. power demand side management and source load forecasting error. 2014 (03).

[4] Wang Xifan, Xiao Yunpeng, Wang Xiuli. Power system under the new situation of supply and demand the interaction between the research and analysis of [J]. Chinese ofthecsee. 2014 (29).

[5] Zhu Qin, Yuan Xiang, the history of finance, Longfei. Active distribution network source / storage / load coordination control technology [J]. East China Electric Power research. 2014 (08).

[6] Yao Jianguo, Yang Shengchun, Wang Ke, Yang Zhenglin, song Xiao Fang. The concept and research framework of "source grid load" interactive operation control in smart grid [J]. power system automation. 2012 (21). 